\title{
Pratiques avicoles et gestion de la fertilité des sols dans les exploitations agricoles de l'Ouest du Burkina Faso
}

\author{
Kalifa Coulibaly', Fernand Sankara1, Salimata Pousga', Philippe J. Nacoulma ${ }^{1}$ et Hassan B. Nacro ${ }^{1}$ \\ 1 Université Nazi Boni (UNB), Institut du Développement Rural (IDR), 01 B.P. 1091 Bobo-Dioulasso 01, Burkina Faso. \\ *Auteur correspondant : kalifacoul1@yahoo.fr
}

Original submitted in on 31st May 2018. Published online at www.m.elewa.org on $31^{\text {st }}$ July 2018 https://dx.doi.org/10.4314/jab.v127i1.2

\section{RÉSUMÉ}

Objectif : l'article montre la place de la volaille dans la production de fumure organique (FO) et la fertilisation des champs des exploitations agricoles (EA).

Méthodologie et résultats : Une enquête d'exploitation agricole a été réalisée avec une approche globale des caractéristiques structurelles et des activités d'élevage de l'exploitation, et la collecte de données déclarées par le chef d'exploitation. L'enquête a été réalisée en 2016 sur un échantillon de 300 EA dans les régions des Cascades et des Hauts-Bassins du Burkina Faso. Les résultats mettent en évidence 5 types d'EA : 2 types d'agriculteurs (type $1: 8,92$ ha, 129 volailles, 4 bovins et 6 petits ruminants ; type $2: 5,48$ ha, 31 volailles, 3 bovins et 7 petits ruminants) et 3 types d'agro-éleveurs (type $3: 10,57$ ha, 42 volailles, 11 bovins et 16 petits ruminants ; type $4: 6,58$ ha, 68 bovins et 26 petits ruminants ; type $5: 36,40$ ha, 117 volailles, 44 bovins, 47 petits ruminants). L'élevage extensif de volaille qui est le système dominant (plus de $70 \%$ des EA enquêtées) apporte près de $26 \%$ du revenu des EA du type 1 . Ce système extensif permet de produire dans l'année 4,67 $\mathrm{kg}$ de fiente par volaille contre 19,21 et 5,08 kg/volaille/an respectivement pour la claustration et le système semi-intensif. La fiente de volaille représente entre 26,46 et $35,72 \%$ de la production de fumure organique et contribue à fertiliser près de $4,50 \%$ des superficies totales cultivées des EA.

Conclusions et application des résultats : Cette contribution de la volaille dans la production de la FO et la fertilisation des champs peut être améliorée en réduisant les pertes durant le processus de production et de collecte des déjections. Le système d'élevage semi-intensif et la claustration se présentent comme des perspectives intéressantes à explorer.

Mots clés : typologie, système d'élevage, volaille, substrat organique, Hauts-Bassins, Cascades.

\begin{abstract}
Use of insects in poultry practices and soil fertility management in farms in Western Burkina Faso Objective : This article shows the place of poultry in the production of organic manure (OM), and the fertilization of farm fields.

Methods and Results: a farm survey was conducted with a holistic approach to the farm's structural characteristics and farming activities, and the collection of data reported by the farm manager. The survey was conducted in 2016 on a sample of 300 agricultural exploitations (AE) in the Cascades and Hauts-Bassins regions of Burkina Faso. The results highlight 5 types of AE: 2 types of farmers (type 1: 8.92 ha, 129 poultry, 4
\end{abstract}


cattle and 6 small ruminants, type 2: 5.48 ha, 31 poultry, 3 cattle and 7 small ruminants) and 3 types of agropastoralists (type 3: 10.57 ha, 42 poultry, 11 cattle and 16 small ruminants, type $4: 6.58$ ha, 68 cattle and 26 small ruminants, type 5: 36,40 ha, 117 poultry, 44 cattle, 47 small ruminants). Extensive poultry farming, which is the dominant system (more than $70 \%$ of the AE surveyed), contributes nearly $26 \%$ of the income of type 1 . This extensive system produces $4.67 \mathrm{~kg}$ of droppings per year per poultry against 19.21 and $5.08 \mathrm{~kg}$ per poultry per year respectively for the intensive and the semi-intensive system. Poultry droppings represent 26.46 to $35.72 \%$ of organic manure production and also contribute to fertilize nearly $4.50 \%$ of the total area cultivated by AE.

Conclusions and application of results : This contribution of poultry in OM production and field fertilization can be improved by reducing losses during the manure production and collection process. The semi-intensive farming system and the cloister are interesting prospects to explore.

Keywords : typology, livestock system, poultry, organic substrate, Hauts-Bassins, Cascades.

\section{INTRODUCTION}

L'amélioration de la fertilité des sols est un passage obligatoire pour la durabilité et la productivité des systèmes de production dans les savanes d'Afrique de l'Ouest (Bationo et al., 2007 ; Koulibaly et al., 2015). Au Burkina Faso, les pratiques culturales basées sur l'exploitation minière des sols, contribuent à la dégradation de leur fertilité (Schipper et Sparling, 2000 ; Sawadogo et al., 2008). Pour faire face à cette dégradation des sols, l'option de fertilisation des cultures est principalement basée sur l'utilisation des fertilisants minéraux associée ou pas à des fertilisants organiques qui sont apportés à des faibles doses. Pourtant les fertilisants organiques sont indispensables pour la restauration de la capacité de production des sols (Bayala et al., 2005; Koulibaly et al., 2015 ; Gomgnimbou et al., 2016). Aujourd'hui, les attentes socio-économiques (alimentation, transport, emploi, éducation, etc.) des populations sont croissantes. Dans un contexte de changements globaux (climatiques, variabilité du prix des intrants, accès au marché), la réponse à ces attentes nécessite le développement de pratiques de gestion durable et résilients des terres. En milieu paysan, il y a une diversité de substrats organiques produits et utilisés par les agriculteurs (Blanchard et al., 2014). Parmi ces substrats organiques, il y a les fientes de volailles. Au Burkina Faso, l'aviculture est fortement pratiquée avec 36419908 têtes et elle est pratiquée tant par les hommes, les femmes que par les enfants (MRA, 2009). L'aviculture occupe une place importante dans les moyens d'existence des populations surtout en milieu rural où les volailles sont élevées non seulement pour la consommation locale, les pratiques rituelles, coutumières, religieuses et sociales, mais aussi pour la génération de revenus à travers la vente (Pousga, 2009). Le poulet local joue donc un rôle très important dans la vie socioculturelle des populations (Fotsa et al., 2007). Malgré son importance socio-économique et nutritionnelle, la volaille est majoritairement élevée en divagation au Burkina Faso (Kondombo, 2005 ; Pousga, 2007 ; Ouédraogo et al., 2015). Par conséquent, une partie importante des déjections de volailles est donc laissée hors de l'exploitation agricole (EA) pendant la divagation. Or, les études de Gomgnimbou et al. (2016) montrent une richesse chimique de la fiente de volaille notamment en azote $(\mathrm{N}=2,58 \%)$ et en phosphore total $(\mathrm{P}=2,73 \%)$ comparativement aux déjections de bovins ( $\mathrm{N}=1,96$ $\%$ et $P=0,96 \%)$, de caprins $(N=1,61 \%$ et $P=1,06$ $\%$ ) et d'ovins ( $N=2 \%$ et $P=0,90 \%)$. Cela laisse voir une certaine efficacité de la fiente de volailles dans l'amélioration de la fertilité du sol et la durabilité des systèmes de productions. Toutefois, avec le système d'élevage extensif dominant en milieu rural, quelle est la quantité de fiente de volailles produite dans les exploitations agricoles ? Quelle peut être la contribution de cette fiente dans la fertilisation des champs? Si des travaux se sont intéressés à la nutrition, la santé et aux aspects socio-économiques de l'aviculture, très peu ont investigué sur le rôle que la volaille peut avoir dans la gestion de la fertilité du sol. Dans le cadre du projet IFWA (Sustainable use of insects to improve livestock production and food security in smallholder farms in West Africa), il est prévu améliorer la nutrition de la volaille par 
l'introduction des asticots. Cela devrait permettre de réduire la divagation de la volaille et par ricochet réduire les pertes de déjections de cette volaille. Le présent article qui s'intéresse à la dimension de la fertilisation du sol par les déjections de volaille, (i)

\section{MATÉRIEL ET MÉTHODES}

Site d'étude : L'étude a été réalisée dans les régions des Hauts-Bassins ( $11^{\circ} 15^{\prime} \mathrm{N}$ et $4^{\circ} 30^{\prime} \mathrm{W}$ ) et des Cascades $\left(10^{\circ} 15^{\prime} \mathrm{N}\right.$ et $\left.4^{\circ} 30^{\prime} \mathrm{W}\right)$ du Burkina Faso (Error! Reference donne les caractéristiques des exploitations agricoles de la zone d'étude, le système d'élevage de volailles qu'elles pratiquent, et (ii) montre la contribution de la volaille dans le revenu des EA et dans la fertilisation de leurs champs.

source not found.) dont Les chefs-lieux sont respectivement Bobo-Dioulasso et Banfora.

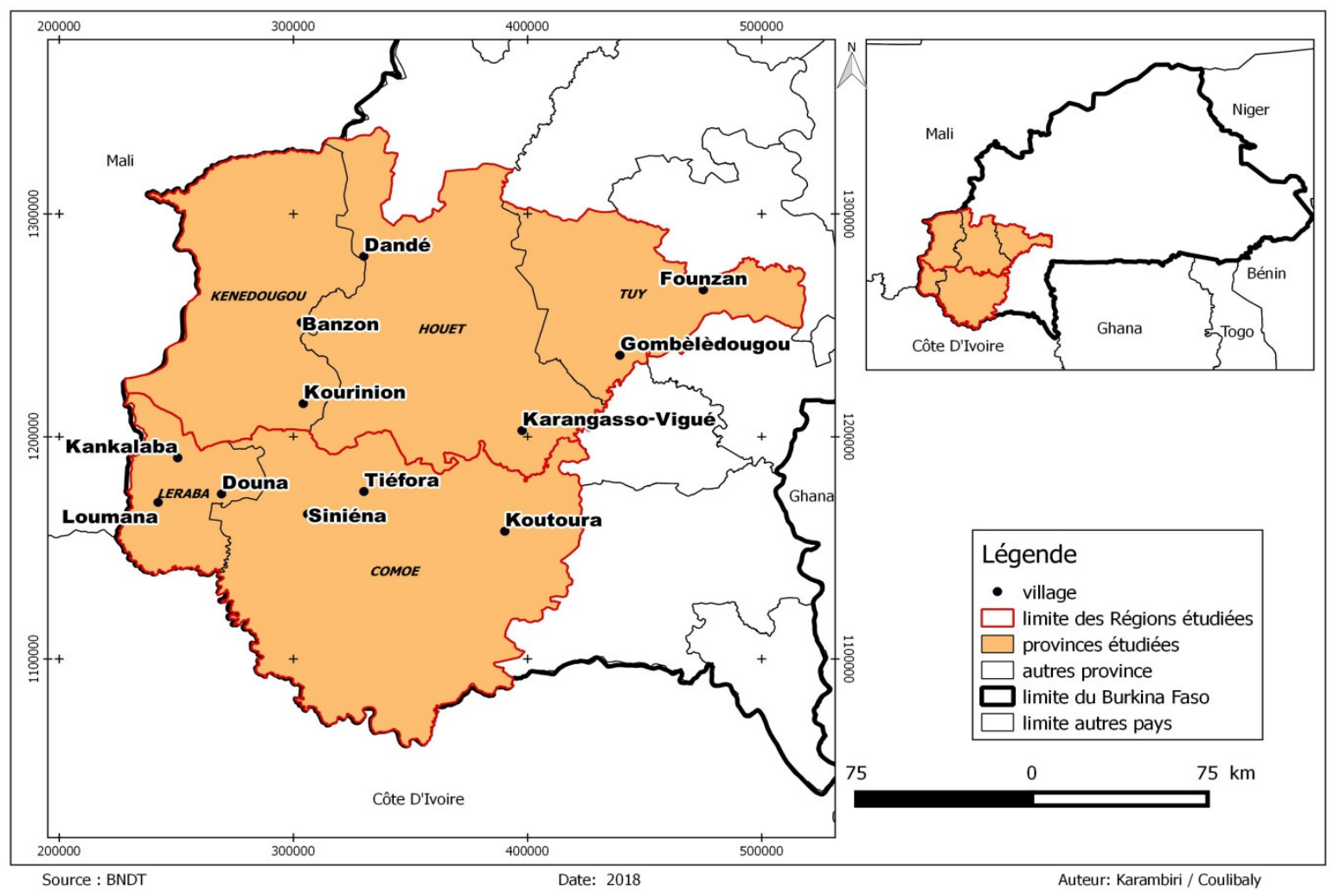

Figure 1 : Carte des villages et régions d'étude

Avec une superficie de $18917 \mathrm{~km} 2$ et une population d'environ 739497 habitants en 2015 (39 habitants/km²), la région des cascades est subdivisée en 2 provinces, 17 communes et 276 villages (INSD, 2016). La production céréalière de cette région était estimée à 182057 tonnes en 2015 contre une production nationale de 4189555 tonnes. L'effectif de la volaille était estimé à 1207387 têtes sur un total de 42220421 têtes au niveau national (INSD, 2016).La région des Hauts-Bassins s'étend sur 25 $606 \mathrm{~km}^{2}$ avec une population estimée à 1961204 habitants en 2015 ( 77 habitants $\left./ \mathrm{km}^{2}\right)$ et repartie sur 3 provinces, 33 communes et 484 villages (INSD, 2016). La production céréalière de cette région était estimée à
861546 tonnes en 2015 et celle de la volaille 5122002 têtes en 2015 (INSD, 2016).

Choix des villages et des exploitations agricoles : Deux (2) villages ont été choisis de façon raisonnée dans chaque province dans la région des Hauts-Bassins. Ainsi, les villages de Karangasso-Vigué et Dandé ont été retenus dans la province du Houet, les villages de Banzon et Kourinion ont été retenus dans la province du Kénédougou et les villages de Gombeledougou et Founzan ont été retenus dans la province du Tuy. Dans la région des Cascades, 3 villages ont été choisis de façon raisonnée dans chaque province. Ce sont les villages de Siniéna, Koutoura et Tiéfora qui ont été retenus dans la 
province de la Comoé et les villages de Douna, Loumana et Kankalaba dans la province de la Léraba. Une enquête a été effectuée auprès de 300 exploitations agricoles (EA), soit 25 EA par village (150 EA par région). Les EA enquêtées ont été retenues par un tirage aléatoire dans chaque village. Les présidents des conseils villageois de développement (CVD) et les chefs des villages ont été associés lors du choix des EA.

Collecte de données : Les données ont été collectées en 2016 par enquête. Pour ce faire, après une préenquête dans le village de Siniéna avec trois (3) chefs d'exploitation agricole. Le questionnaire final qui a été administré aux chefs d'exploitations agricoles de l'échantillon d'enquête, portait sur la taille, les actifs, l'élevage, les surfaces cultivées, le revenu, l'utilisation d'insectes ou de larves d'insectes dans l'alimentation de la volaille, la production et l'utilisation de substrats organiques des exploitations agricoles. Le revenu de l'exploitation a été estimé par an à partir de tout ce qui lui permet de faire des rentrées d'argent. Le revenu avicole a été obtenu à partir du nombre de têtes vendues par an et du prix moyen de vente par tête. Les quantités de substrats organiques (fiente de volaille, déjections de bovins, d'ovins et e caprins) ont été estimées en faisant appel à la mémoire de l'enquêté qui donnait la fréquence de collecte et le récipient utilisé pour la collecte. L'enquête qui se déroulait en langue dioula, durait environ 1 heure de temps. Les difficultés rencontrées durant l'enquête, sont surtout liées à l'estimation exacte des quantités des substrats organiques et du nombre de volailles vendues.

Analyse de données

\section{RÉSULTATS}

Diversité des exploitations agricoles: La Error! Reference source not found. montre que le plan factoriel, constitué par les axes F1 et F2, explique 45,19 $\%$ de la variabilité. Le cosinus carré des variables montre que c'est seulement l'axe F1 qui discrimine les exploitations agricoles (EA) en fonction de la surface totale des champs, du nombre de personnes, du nombre d'actifs et du nombre d'animaux (ovins, caprins et bovins) (Tableau 2). A partir du tableau des coordonnées des observations de ces axes factoriels obtenus de l'ACP, une classification ascendante hiérarchique $(\mathrm{CAH})$ a été réalisée. Elle donne 5 types d'EA avec une coupure du dendrogramme à un niveau de dissimilarité qui est de 160,39 (Error! Reference source not found.). L'analyse des caractéristiques de ces types d'EA (Tableau 3),
Analyse de la diversité des EA : La méthode d'analyse multi-variée a été utilisée pour réaliser une typologie des exploitations agricoles (EA). Une analyse en composantes principales (ACP) a été effectuée sur 8 variables (Tableau 1). Une classification ascendante hiérarchique (CAH) a ensuite été réalisée à partir du tableau des coordonnées des observations sur les axes factoriels obtenus de l'ACP. Elle a permis de constituer des groupes homogènes d'EA (types). Le logiciel XLSTAT 2015.4.01 a été utilisé pour l'analyse multivariée.

Détermination de la capacité de fertilisation des champs par les EA : La proportion de la surface de champs que peut fertiliser les EA de chaque type a été déterminée à partir de la formule suivante que nous avons établie :

CFC (\%) $=\frac{\text { Qt } 80 * \text { STC }}{2000} \times 100($ CFC = capacité de fertilisation des champs; Qt SO = quantité de substrats organiques $(\mathrm{kg})$; STC $=$ surface totale des champs (ha) et 2000 = quantité de substrats organiques à apporter sur 1 ha, exprimés en $\mathrm{kg} / \mathrm{ha}$ ). La dose de $2000 \mathrm{~kg} / \mathrm{ha}$ a été retenue sur la base des travaux de Berger (1996) et de Koulibaly et al. (2015) qui indiquent que cette dose est nécessaire chaque année pour maintenir la productivité des systèmes de cultures.

Réalisation des figures et tableaux: Les figures et tableaux ont été générés à l'aide du Microsoft Excel 2010. Au regard de la difficulté de collecte de certaines données auprès des personnes enquêtées, le nombre d'EA varie pour un type donné d'une variable à une autre.

montre que le type 5 (5 EA) regroupe les EA qui ont de grandes superficies cultivées $(36,40$ ha), de grands nombres de personnes ( 42 personnes), d'actifs (28 actifs agricoles et 9 actifs pour l'élevage), de caprins (27 têtes) et de poulets (115 têtes). Le type 4 (18 EA) se caractérise par un grand nombre d'ovins (26 têtes) et de bovins (68 têtes). Le type 3 (52 EA) vient après le type 5 en termes de surface de champs cultivés (10,57 ha), de nombre de personnes (24 personnes) et d'actifs pour l'élevage (8 actifs). Le type 1 (39 EA) se caractérise surtout par le nombre total de volailles ( 109 poulets et 21 pintades). Le type 2 (186 EA) regroupe le grand nombre d'EA ayant de petites superficies cultivées (5,48 ha) avec peu de personnes, d'actifs et d'animaux.

Tableau 1 : Liste des variables utilisées pour l'analyse multi-variée 
Kalifa et al, J. Appl. Biosci. 2018 Pratiques avicoles et gestion de la fertilité des sols dans les exploitations agricoles de l'Ouest du Burkina Faso

\begin{tabular}{l|l|l}
\hline Variables & Unité & Description \\
\hline STC & ha & surface totale des champs \\
Nb personnes & numérique & nombre de personnes \\
Nb actifs agricole & numérique & nombre d'actifs agricoles \\
Nb actifs élevage & numérique & nombre d'actifs pour élevage \\
Nb années PEV & année & nombre d'années de pratique d'élevage de volailles \\
Nb Ovins & numérique & nombre d'ovins \\
Nb Caprins & numérique & nombre de caprins \\
Nb Bovins & numérique & nombre de bovins \\
Nb Poulets & numérique & nombre de poulets \\
Nb Pintades & numérique & nombre de pintades \\
\hline
\end{tabular}

Tableau 2 : Cosinus carré des variables issues de l'ACP

\begin{tabular}{l|c|c}
\hline Variables & F1 & F2 \\
\hline STC (ha) & $\mathbf{0 , 5 0 0}$ & 0,004 \\
Nb personnes & $\mathbf{0 , 6 9 2}$ & 0,064 \\
Nb actifs agricole & $\mathbf{0 , 5 9 9}$ & 0,182 \\
Nb actifs élevage & $\mathbf{0 , 2 6 0}$ & 0,173 \\
Nb années PEV & 0,072 & 0,031 \\
Nb Ovins & 0,345 & 0,179 \\
Nb Caprins & 0,291 & 0,186 \\
Nb Bovins & 0,269 & 0,238 \\
Nb Poulets & 0,073 & 0,101 \\
Nb Pintades & 0,002 & 0,257 \\
\hline
\end{tabular}

Les valeurs en gras correspondent pour chaque variable au facteur pour lequel le cosinus carré est le plus grand $\mathrm{STC}=$ surface totale des champs $; \mathrm{Nb}=$ nombre $; \mathrm{PEV}=$ élevage de volailles

Tableau 3 : Caractéristiques des différents types d'exploitations agricoles

\begin{tabular}{l|c|c|c|c|c}
\hline & Type 1 & Type 2 & Type 3 & Type 4 & Type 5 \\
\hline Nb EA & 39 & 186 & 52 & 18 & 5 \\
\hline STC (ha) & 8,92 & 5,48 & 10,57 & 6,58 & 36,40 \\
Nb personnes & 11,33 & 10,88 & 23,85 & 15,56 & 42,00 \\
Nb actifs agricole & 6,54 & 6,13 & 14,50 & 7,44 & 28,20 \\
Nb actifs élevage & 3,41 & 2,64 & 7,77 & 3,00 & 9,00 \\
Nb année PEV & 25,26 & 21,01 & 22,65 & 17,89 & 33,80 \\
Nb Ovins & 6,38 & 3,51 & 8,23 & 25,78 & 20,00 \\
Nb Caprins & 5,97 & 3,52 & 8,54 & 20,17 & 27,00 \\
Nb Bovins & 4,64 & 3,15 & 11,31 & 68,28 & 44,40 \\
Nb Poulets & 108,64 & 30,31 & 42,54 & 55,33 & 115,00 \\
Nb Pintades & 20,97 & 1,15 & 4,37 & 8,67 & 2,00 \\
\hline
\end{tabular}

$E A=$ exploitation agricoles, $S T C=$ surface totale des champs $; \mathrm{Nb}=$ nombre $; P E V=$ élevage de volailles 


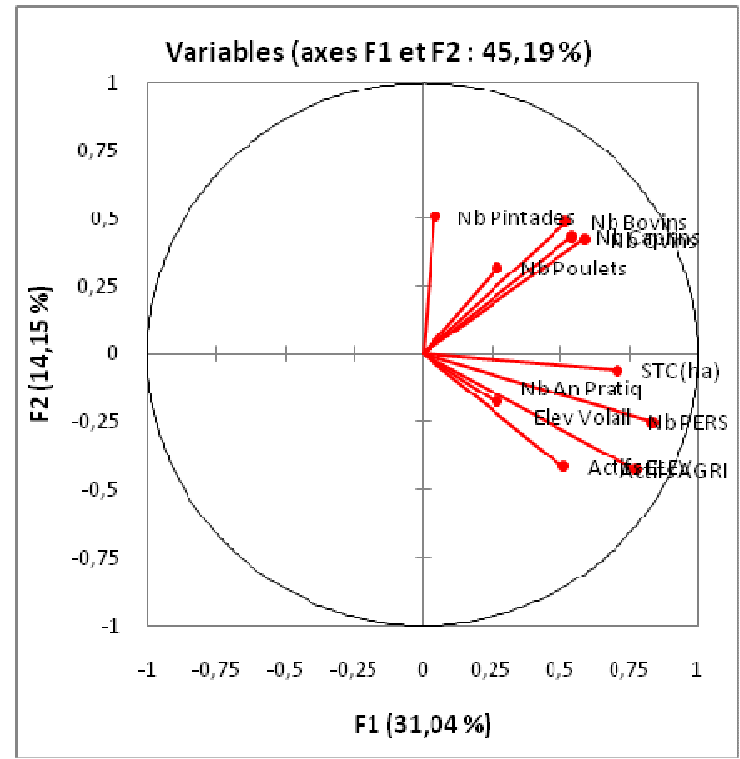

Figure 2 : Projection des variables sur un plan constitué par les deux premiers facteurs (F1 et F2)

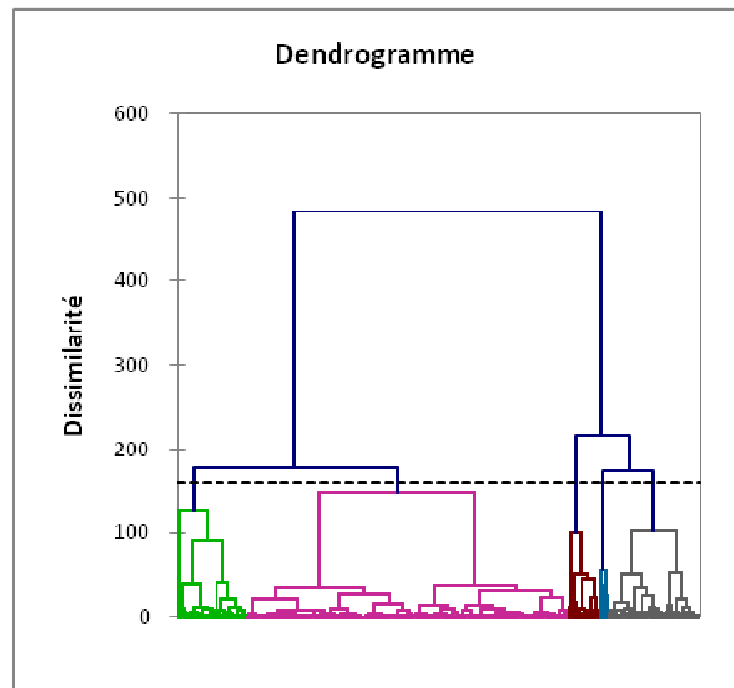

Figure 3 : Classification ascendante hiérarchique des pratiques et des conditions pluviométriques (Dendrogramme)

Systèmes d'élevage aviaire des exploitations agricoles : La majorité des EA enquêtées $(n=224)$ pratiquent l'élevage extensif avec un nombre faible de volailles (43 poulets et 5 pintades) comparativement à celles qui pratiquent la claustration (130 poulets et 3 pintades) et l'élevage semi-intensif (61 poulets et 4 pintades) (Error! Reference source not found.). Le nombre d'EA est de 5 et 53 respectivement pour la claustration et le système semi-intensif. 


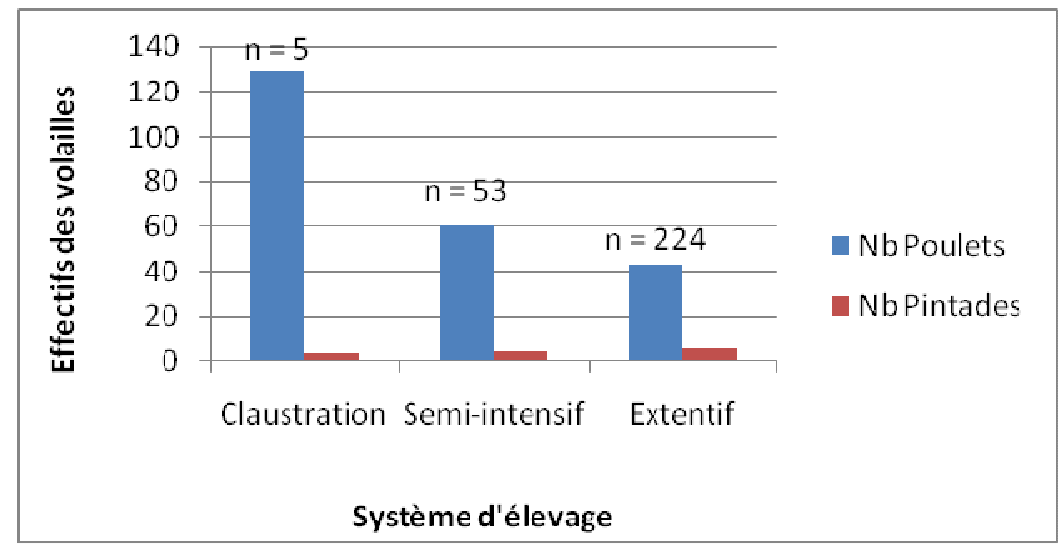

Figure 4 : Effectifs de volaille suivant le système d'élevage $(\mathrm{Nb}=$ nombre $)$

Le système extensif d'élevage de volaille est le plus dominant dans tous les types d'EA (Error! Reference source not found.). Il est pratiqué par plus de $60 \%$ des EA quel que soit le type d'EA. Le système extensif est suivi dans l'ordre par le système semi-intensif et la claustration. Moins de $30 \%$ des EA pratiquent le système semi-intensif excepté le type 5 qui a enregistré $40 \%$. Dans le type 4 où près de $95 \%$ des EA pratiquent le système extensif, le système semi-intensif n'est pas pratiqué. L'élevage de volaille par claustration n'est pas pratiqué par les EA du type 5 et la proportion la plus élevée est notée dans le type 4 avec près de $6 \%$ des EA.

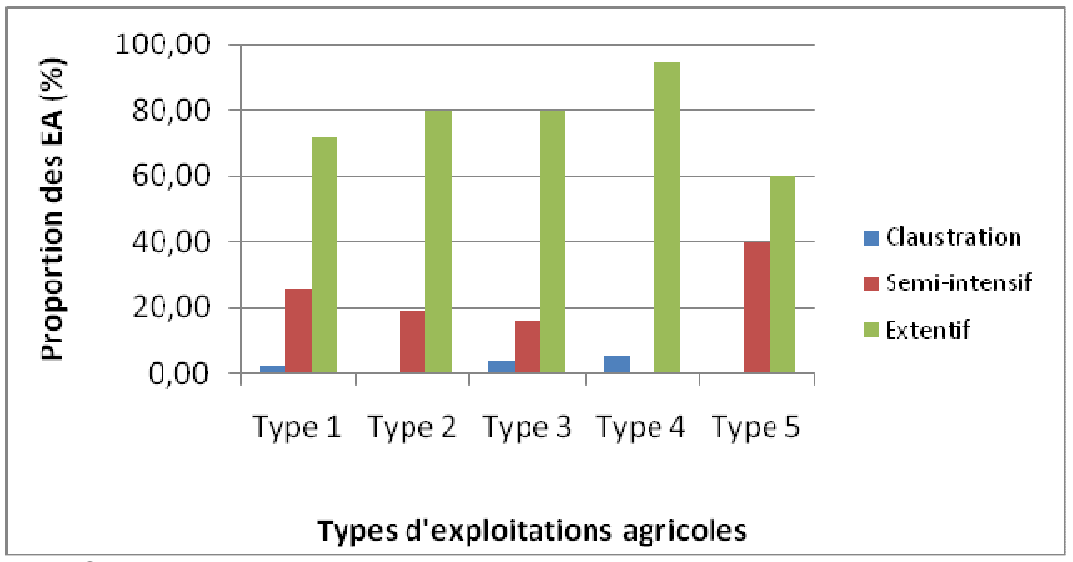

Figure 5 : Systèmes d'élevage de volaille pratiqué par type d'exploitations agricoles (EA)

Infrastructures avicoles des exploitations agricoles : Les données sur les infrastructures avicoles montrent que plus de $50 \%$ des EA quel que soit le type, disposent d'un poulailler, d'une poussinière, d'une mangeoire, d'un abreuvoir, d'un pondoir et d'un perchoir (). Pour le poulailler, la proportion des EA qui en disposent dépasse $80 \%$ pour tous les types d'EA et atteigne $97 \%$ pour le type 1. En revanche, le perchoir est parfois utilisé par moins de $50 \%$ des EA du type $2(48,90 \%)$ et du type 3 $(49,02 \%)$.

Utilisation d'insectes ou de larves d'insectes comme source protéique dans l'aviculture : La proportion des
EA qui produisent et utilisent les asticots dans la nutrition aviaire, varie entre $3,92 \%$ (type 3) et $20 \%$ (type 5) (Tableau 5). Quel que soit le type d'EA, près de $50 \%$ des EA utilisent toujours des termites dans la nutrition de leur volaille (Error! Reference source not found.). On note que cette proportion atteigne $60 \%$ des EA au niveau du type 5 . A l'inverse, plus de $80 \%$ des EA quel que soit le type d'EA, n'utilisent jamais les larves d'autres insectes (Error! Reference source not found.). Seulement $20 \%$ des EA du type 5 utilisent parfois les larves d'autres insectes. 
Kalifa et al, J. Appl. Biosci. 2018 Pratiques avicoles et gestion de la fertilité des sols dans les exploitations agricoles de l'Ouest du Burkina Faso

Tableau 4 : Proportion des EA utilisant des infrastructures avicoles

\begin{tabular}{|l|l|c|c|c|c|c|c}
\hline & & Poulailler & Poussinière & Mangeoire & Abreuvoir & Pondoir & Perchoir \\
\hline \multirow{2}{*}{ Type 1 $(\mathrm{n}=39)$} & Non & 2,56 & 35,90 & 41,03 & 20,51 & 33,33 & 38,46 \\
& Oui & 97,44 & 64,10 & 58,97 & 79,49 & 66,67 & 61,54 \\
\hline \multirow{2}{*}{ Type 2 $(\mathrm{n}=182)$} & Non & 14,29 & 41,76 & 46,15 & 30,77 & 40,88 & 51,10 \\
& Oui & 85,71 & 58,24 & 53,85 & 69,23 & 59,12 & 48,90 \\
\hline \multirow{2}{*}{ Type 3 $(\mathrm{n}=51)$} & Non & 13,73 & 37,25 & 49,02 & 25,49 & 39,22 & 50,98 \\
& Oui & 86,27 & 62,75 & 50,98 & 74,51 & 60,78 & 49,02 \\
\hline \multirow{2}{*}{ Type 4 $(\mathrm{n}=18)$} & Non & 11,11 & 33,33 & 16,67 & 5,56 & 22,22 & 38,89 \\
& Oui & 88,89 & 66,67 & 83,33 & 94,44 & 77,78 & 61,11 \\
\hline \multirow{2}{*}{ Type 5 $(\mathrm{n}=5)$} & Non & 20,00 & 20,00 & 40,00 & 0,00 & 20,00 & 40,00 \\
& Oui & 80,00 & 80,00 & 60,00 & 100,00 & 80,00 & 60,00 \\
\hline
\end{tabular}

Tableau 5 : Production et utilisation d'asticots dans la nutrition aviaire

\begin{tabular}{|l|c|c|c|c|c|c}
\hline & $\begin{array}{c}\text { Type 1 } \\
(\mathrm{n}=39)\end{array}$ & $\begin{array}{c}\text { Type 2 } \\
(\mathrm{n}=174)\end{array}$ & $\begin{array}{c}\text { Type 3 } \\
(\mathrm{n}=51)\end{array}$ & $\begin{array}{c}\text { Type 4 } \\
(\mathrm{n}=18)\end{array}$ & $\begin{array}{c}\text { Type 5 } \\
(\mathrm{n}=5)\end{array}$ \\
\hline Utilisation & Non & 94,87 & 92,53 & 96,08 & 88,89 & 80 \\
Asticots & Oui & 5,13 & 7,47 & 3,92 & 11,11 & 20 \\
\hline Production & Non & 94,87 & 93,68 & 96,08 & 88,89 & 80 \\
Asticots & Oui & 5,13 & 6,32 & 3,92 & 11,11 & 20 \\
\hline
\end{tabular}

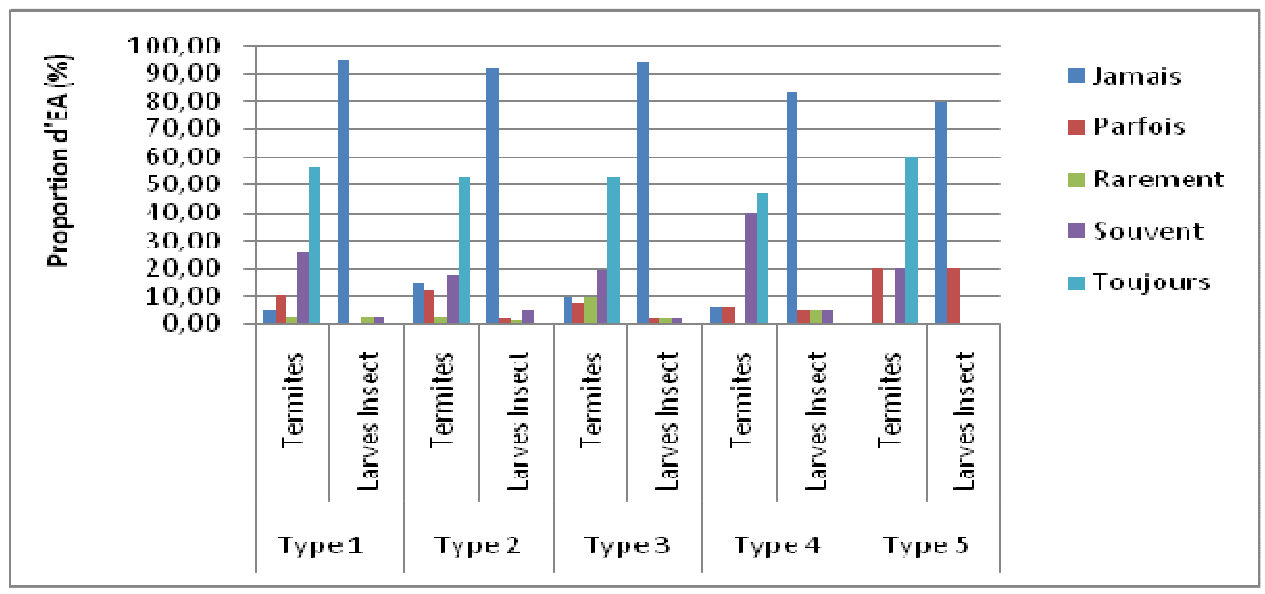

Figure 6 : Utilisation de termites et d'autres larves d'insectes dans la nutrition aviaire

Contribution du revenu avicole dans le revenu total des exploitations agricoles : La contribution du revenu engendré par l'aviculture dans le revenu global des EA est plus importante pour le type 1 (25,80 \%) comparativement au type $2(14,63 \%)$, type $3(14,75 \%)$, type $4(15,62 \%)$ et type $5(6,61 \%)$ (Error! Reference source not found.). 


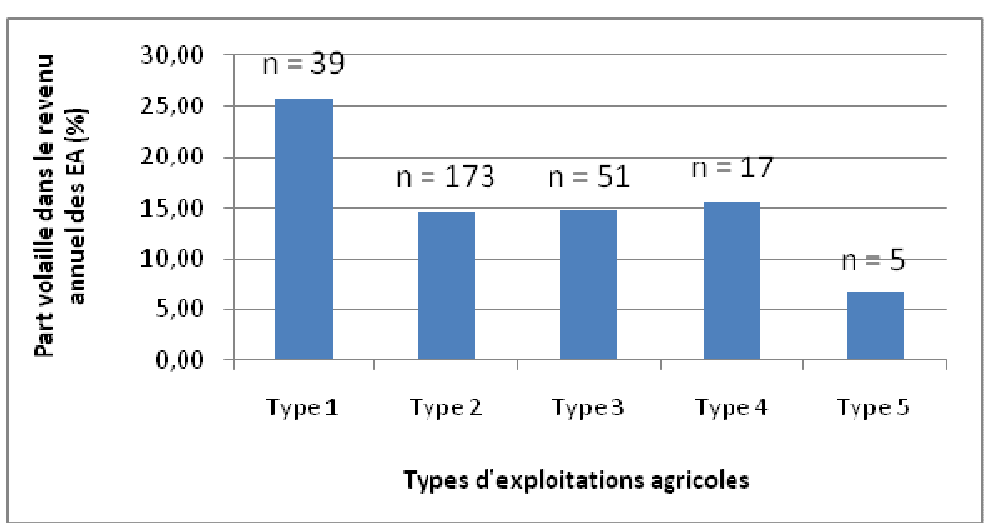

Figure 7 : Proportion du revenu avicole dans le revenu total des EA

Production de substrats organiques dans les exploitations agricoles: La Figure 8 montre que le système d'élevage par claustration permet d'avoir beaucoup de litière de volailles (2555 kg/an) par rapport au système extensif (330 kg/an) et semi-intensif (224 kg/an). En rapportant par tête de volaille selon le système d'élevage, cela donne une production annuelle de 19,21 $\mathrm{kg} / \mathrm{sujet}$ pour la claustration et de $5,08 \mathrm{~kg} / \mathrm{sujet}$ pour le système semi-intensif et $4,67 \mathrm{~kg} / \mathrm{sujet}$ pour le système extensif.

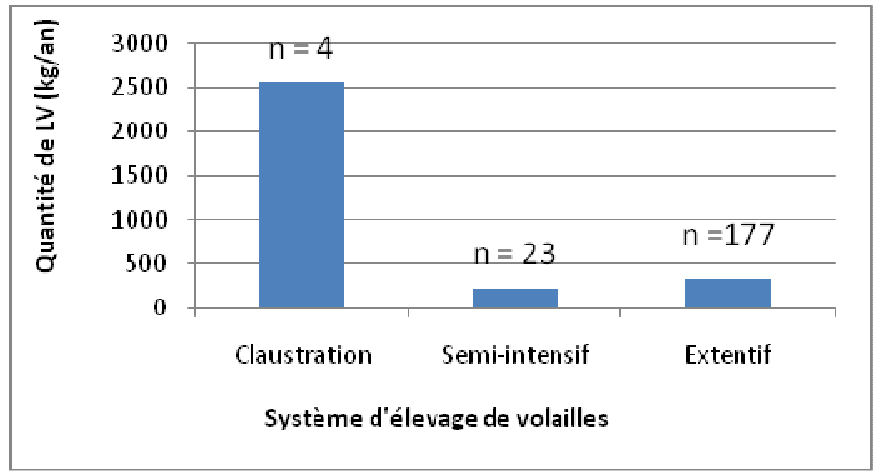

Figure 8 : Variation de la quantité de litière de volailles (LV) selon le système d'élevage

La production de litière de volailles est plus importante dans les EA du type 1 (800 kg/an) comparativement aux EA du type 2 (196 kg/an), du type 3 (414 kg/an), du type
4 (415 kg/an) et du type 5 (375 kg/an) (Figure 9). En rapportant par tête de volaille, cela donne 6,$16 ; 6,32$; 9,$41 ; 6,48$ et $3,21 \mathrm{~kg} / \mathrm{sujet}$.

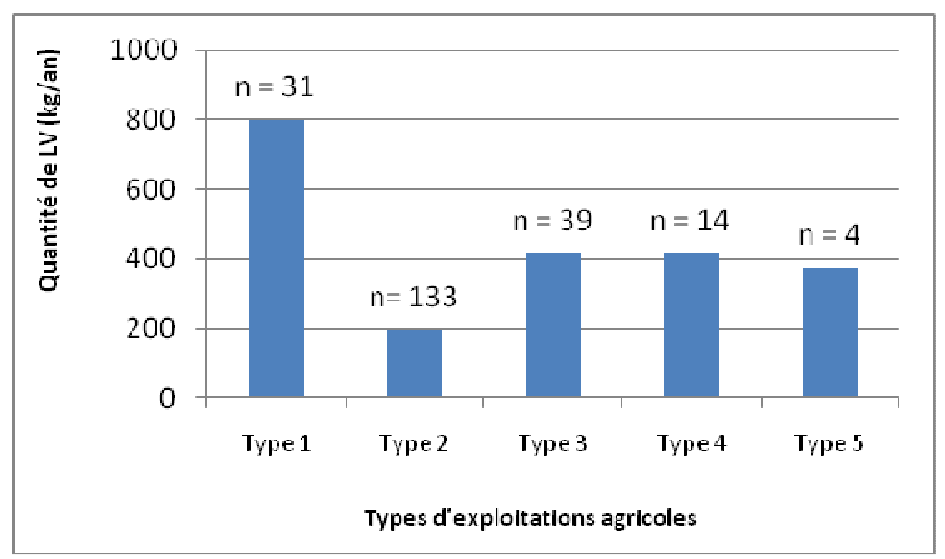

Figure 9 : Variation de la quantité de litière de volailles (LV) selon les types d'exploitation agricole 
La Figure 10 montre que les EA de type 5 ont une forte capacité de production de substrats organiques (SO) avec des quantités pouvant atteindre $12166 \mathrm{~kg} / \mathrm{an}$. Elles sont suivies par des EA de type 4 avec $9366 \mathrm{~kg} / \mathrm{an}$. La plus faible production de $\mathrm{SO}$ est réalisée par les EA du type 2 (1 $365 \mathrm{~kg} / \mathrm{an})$. On observe que la contribution de la litière de volailles dans la production de $\mathrm{SO}$ varie d'un type d'EA à un autre avec 35,72 \%, 33,65\%, 26,46\%, $30,77 \%$ et $30,39 \%$ respectivement pour les types $1,2,3$, 4 et 5 (Figure 11).

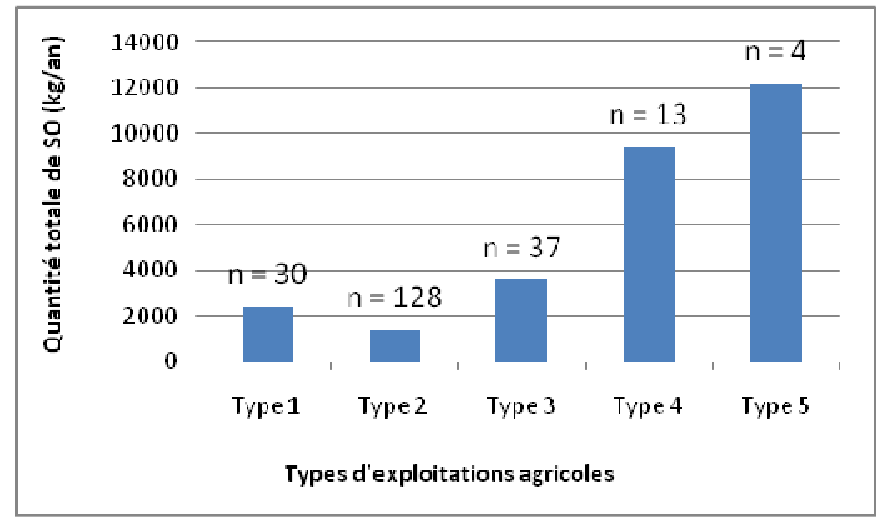

Figure 10 : Variation de la quantité de substrats organiques (SO) selon les types d'exploitation agricole

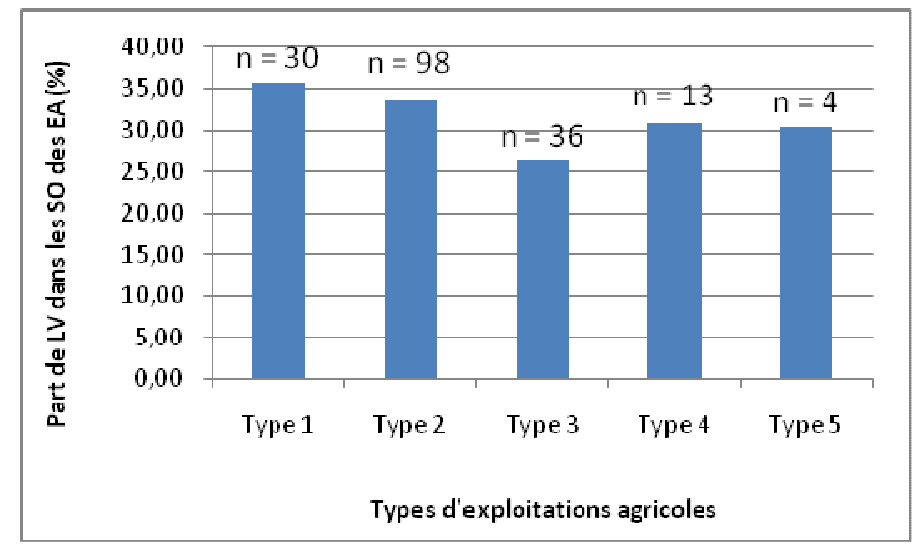

Figure 11 : Contribution de la litière de volailles dans la production de substrats organiques (SO) selon les types d'exploitation agricole $(\mathrm{EA})$

Cultures bénéficiant de substrats organiques produits dans les exploitations agricoles : Le Tableau 6 montre qu'entre 45 et $60 \%$ des EA des différents types d'EA affirment qu'elles appliquent les SO sur toutes les cultures sans exception. Toutefois, il ressort que pour tous les types d'EA, une proportion importante des EA (entre 33 et $46 \%$ ) appliquent les SO sur le maïs.

Capacité de fertilisation organique des champs par les exploitations agricoles: Les EA du type 1 se présentent comme celles pouvant fertiliser une portion importante $(4,48 \%)$ de la superficie totale de leur champ (STC) avec de la litière de volailles (Figure 12). Elles sont suivies par les EA du type 4 (3,15\% de STC), celles du type 3 ( $1,95 \%$ de STC) et du type 2 (1,79\% de STC). Les EA du type 5 ont une faible capacité de fertilisation de leur champ $(0,52 \%)$ avec la litière de volailles. La Figure 13 montre que ce sont les EA du type 4 qui peuvent fertiliser une proportion importante $(71,13 \%)$ de la STC avec les substrats organiques issus de l'ensemble de leur élevage. Elles sont suivies par les EA de type 3 $(16,97 \%$ de STC) et de type 5 (16,71 \% de STC). Les EA des types 1 et 2 ne peuvent fertiliser que 13,59 et 12,44 $\%$ de la surface totale de leur champ avec les substrats organiques produites sur leurs exploitations. 
Kalifa et al, J. Appl. Biosci. 2018 Pratiques avicoles et gestion de la fertilité des sols dans les exploitations agricoles de l'Ouest du Burkina Faso

Tableau 6 : Les cultures bénéficiant de fertilisation organique dans les EA

\begin{tabular}{|c|c|c|}
\hline Types d'exploitations agricoles (EA) & Cultures & Proportion (\%) \\
\hline \multirow{5}{*}{ Type $1(n=37)$} & Maïs & 45,95 \\
\hline & Maraîchage & 2,70 \\
\hline & Riz & 2,70 \\
\hline & Toutes les cultures & 45,95 \\
\hline & Verger & 2,70 \\
\hline \multirow{8}{*}{ Type $2(n=149)$} & Maïs & 43,62 \\
\hline & Maraîchage & 2,68 \\
\hline & Pépinières & 0,67 \\
\hline & Riz & 0,67 \\
\hline & sésame & 0,67 \\
\hline & Sorgho & 3,36 \\
\hline & Toutes les cultures & 47,65 \\
\hline & Verger & 0,67 \\
\hline \multirow{4}{*}{ Type $3(n=50)$} & Maïs & 34,00 \\
\hline & Maraîchage & 6,00 \\
\hline & Sorgho & 6,00 \\
\hline & Toutes les cultures & 54,00 \\
\hline \multirow{3}{*}{ Type 4 ( $n=18)$} & Maïs & 33,33 \\
\hline & Sorgho & 11,11 \\
\hline & Toutes les cultures & 55,56 \\
\hline \multirow{2}{*}{ Type $5(n=5)$} & Maïs & 40 \\
\hline & Toutes les cultures & 60 \\
\hline
\end{tabular}

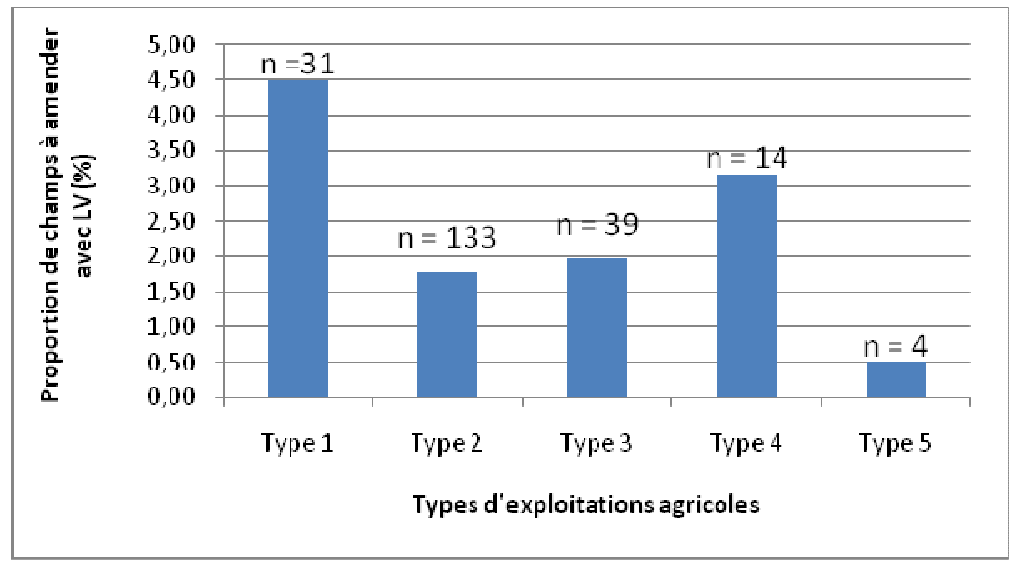

Figure 12 : Estimation de la capacité d'amendement des terres des EA à partir de la litières de volailles (LV) 


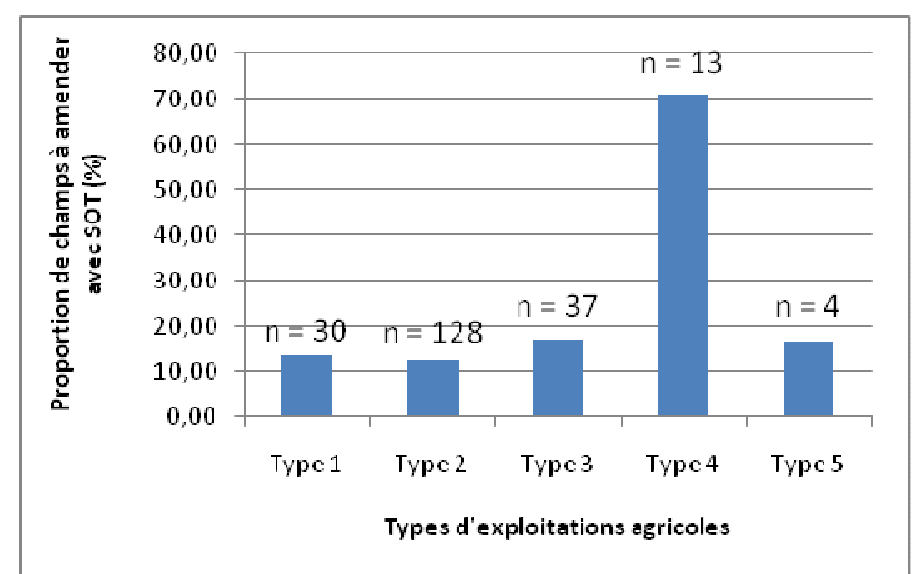

Figure 13 : Estimation de la capacité d'amendement des terres des EA à partir de SO

\section{DISCUSSION}

De façon globale, on note une pratique simultanée de l'agriculture et de l'élevage dans toutes les EA et ce, à des degrés divers. Selon Blanchard et al. (2011), cette intégration agriculture-élevage répond à un impératif de sécurisation économique. Les résultats montrent que c'est la superficie totale des champs cultivés, le nombre de personnes, le nombre de bovins et de volailles qui sont les facteurs discriminants des EA. Selon la typologie élaborée par Vall et al. (2006) basée sur la superficie cultivée et la taille du troupeau de bovins, on peut dire que les EA de type 1 et de type 2 sont des agriculteurs car elles disposent de moins de 10 bovins. Dans ce groupe d'agriculteurs, on pourrait dire que les EA du type 1 (13\% des EA enquêtées) diversifient leurs productions animales avec un accent sur l'aviculture comparativement au type 2 qui regroupe $62 \%$ des EA enquêtées. Selon toujours la typologie de Vall et al. (2006), les EA de type 3,4 et 5 sont des agro-éleveurs du fait qu'elles possèdent plus de 10 bovins et cultivent plus de 5 ha. De même, les EA du type 5 (2 \% des EA enquêtées) sont des grands agro-éleveurs avec une plus grande surface cultivée, un développement important de l'aviculture et un nombre important d'actifs agricoles comparativement aux deux autres types. Les EA du type 4 (6\% des EA enquêtées) sont des éleveur-pasteurs qui se sédentarisent de plus en plus au Burkina Faso. L'analyse du système d'élevage de volailles montre une prédominance de l'élevage extensif par rapport à la claustration et au système semi-intensif. Ce résultat confirme ceux de Ouédraogo et al. (2015) qui montrent que la volaille est élevée en divagation au Burkina Faso. Toutefois, il faut noter que plus de $80 \%$ des EA de chaque type disposent d'un poulailler. Cela indique que la volaille passe la nuit dans les poulaillers et c'est le jour qu'elle est livrée à la divagation. Cette divagation est certes source de déperdition de déjection et de dépense d'énergie, mais elle permet à la volaille de couvrir ses besoins en protéines, en éléments minéraux et en vitamines. On note aussi que les EA passent de l'extensif au semi-intensif ou à la claustration dès lors que la taille de la volaille est importante. Si ces systèmes d'élevage peuvent permettre d'accroître la productivité de la volaille, il reste entendu qu'ils vont exiger un apport régulier d'aliments protéiques et riches en éléments minéraux et vitamines, donc un apport significatif d'intrants de production (Pousga, 2007). Concernant l'utilisation d'insectes et de leurs larves dans la nutrition aviaire, on note que la majorité des EA utilise les termites et très peu d'EA utilisent les larves d'insecte. Cela peut s'expliquer par le fait que l'utilisation des termites dans la nutrition de la volaille est une pratique traditionnelle qui se perpétue de génération en génération. En effet, l'approvisionnement en aliments qualitatifs pour l'alimentation de la volaille est bien souvent le principal problème rencontré par les éleveurs et surtout les petits exploitants agricoles en Afrique de l'ouest (Hardouin, 1986). En aviculture traditionnelle, les termites occupent une place très importante. Ils sont utilisés par de nombreux éleveurs pour complémenter leur volaille, essentiellement composé de poulets et de pintades. Au Burkina Faso, les termites viennent en complément aux céréales et près de $70 \%$ de petits producteurs les utilisent (Dao, 2016 ; Ouedraogo, 2016). Ils sont disponibles et utilisés durant toutes les saisons de l'année (Diawara; 2013). Cependant, ces aliments sont apportés en quantités infimes si bien que la volaille cherche ellemême le reste de leurs rations avec les invertébrés, les autres insectes ou larves d'insectes tel que les larves de mouches ou asticots en rodant autour des concessions. Ces asticots constituent aussi une source importante de protéines animales pour l'aviculture traditionnelle. 
Cependant, leur production et leur utilisation sont très limitées à un faible nombre d'aviculteurs au Burkina Faso et ceci serait lié à la méconnaissance des avantages liés à l'utilisation des asticots comme source protéinique (Hardouin, 2003). A cela s'ajoute les contraintes liées à la production qui sont entre autres les odeurs qui dérangent le voisinage, des habitudes alimentaires de la volaille dictées par des services d'élevage, l'aspect répugnant des asticots pour l'aviculteur. Une sensibilisation sur l'opportunité à saisir permettrait aux producteurs d'améliorer la productivité et la compétitivité de l'aviculture traditionnelle. La contribution de l'aviculture dans le revenu des EA est plus importante chez le type 1 (plus de $25 \%$ du revenu total) comparativement aux autres types d'EA. Les données des EA du type 5 montrent que malgré une taille importante de volaille, la part du revenu avicole dans le revenu global devient faible lorsque les surfaces cultivées sont grandes et le nombre de bovins élevés est important. On pourrait donc comprendre que l'aviculture est un moyen de sécurisation économique qui peut avoir toute son importance chez les petits agriculteurs qui auraient des difficultés à s'investir dans l'élevage de ruminants et particulièrement de bovins. En effet, l'aviculture avait été utilisée dans plusieurs projets de développement en Afrique et en Asie pour lutter contre la pauvreté en milieu rural à cause du fait qu'elle est considérée comme « l'oiseau des pauvres » : c'est -à-dire l'animal le moins cher que n'importe quelle personne peut élever y compris les femmes, les jeunes, les vieillards et même ceux qui n'ont pas de terre (FAO, 2002). Par ailleurs, le poulet local joue un rôle important dans la vie socioculturelle des populations pour des cérémonies de mariage, le rejet de la malédiction, la pharmacopée traditionnelle et le maintien de la cohésion sociale au sein des communautés traditionnelles à travers des dons et la réception des visiteurs de marque (Fotsa et al., 2007 ; Pomalegni et al., 2016). Les résultats sur la production de substrats organiques montrent que la claustration permet de produire plus de litière de volaille par rapport au système extensif et semi-intensif. La divagation serait donc un système qui favorise une déperdition importante des déjections de volaille pour les EA. On observe par

\section{CONCLUSION}

Le présent papier montre qu'il y a une diversité d'exploitations agricoles qui intègrent l'agriculture et l'élevage dans la zone d'étude. La taille de la surface cultivée et le nombre d'animaux donnent le degré d'intégration de ces deux activités. II ressort aussi que l'intégration de l'aviculture à l'agriculture prend une ailleurs que la quantité de litière produite n'est pas liée à la taille de la volaille suivant les types EA. On pourrait expliquer par l'importance accordée à la gestion de la volaille. Les EA du type 1 qui ont une taille importante de volailles, produisent plus de litière comparée aux EA de type 5 qui en produisent moins avec à peu près le même nombre de volaille. Les EA du type 5 accorderaient une importance à l'élevage de bovins qui produisent suffisamment de déjections par rapport à la volaille. Les 3 piliers de l'intégration agriculture-élevage sont la traction animale, la production de fumure organique, et la production de fourrage (Dugué 1989 ; Landais et Lhoste, 1990 ; Lhoste, 2004). La plus part des travaux sur l'intégration agriculture-élevage ont mis l'accent sur l'élevage de gros animaux qui peuvent non seulement assurer le travail agricole (labour, transport) mais produire beaucoup de fumier au niveau des étables et des parcs améliorés (Berger et al., 1987 ; Zoumana et al., 1994). La volaille n'a donc pas été suffisamment prise en compte dans les travaux sur l'intégration agriculture-élevage. Or, les résultats de cette étude montrent que la volaille peut contribuer à près de $30 \%$ de la production des substrats organiques dans les EA. Elle peut permettre également aux EA qui intègrent fortement l'aviculture à l'agriculture, de fertiliser près de $5 \%$ de leur champ contre $0,52 \%$ pour les agro-éleveurs qui intègrent plus le bétail à l'agriculture. En revanche, ces EA qui sont dominées par l'élevage de bétail présentent une plus grande capacité de fertilisation de leur champ (plus de $70 \%$ des superficies cultivées) avec l'ensemble des substrats organiques qu'elles produisent. La couverture des besoins des autres EA en substrats organiques varie entre 12 et $17 \%$ selon l'importance du bétail. Cela est en accord avec les données de Vall et al. (2011) qui ont obtenu entre 7 et $28 \%$ de la couverture des besoins des exploitations en fumure organique. Du reste, la production de substrats organiques sur les EA reste modeste et les apports se font dans la majorité des EA sur toutes les cultures sans préférence. On pourrait donc dire que le niveau de dégradation des terres est très déterminant dans le choix de la fertilisation que le type de culture qui dévrait en bénéficier.

proportion importante dans certains EA disposant d'un nombre réduit de bétail (cas du type 1). Cela permet à ces EA d'avoir un revenu avicole qui représente près de $26 \%$ de leur revenu global. On note également que la litière de volaille peut contribuer significativement à la production de FO et à la fertilisation des sols de ces EA 
malgré la dominance de la pratique de l'élevage extensif. Pour accroitre la production de la litière de volaille en particulier et de la fumure organique en générale, et contribuer à relever la fertilité des sols et améliorer la productivité des cultures par des apports significatifs de fumure, il faut estimer les pertes de déjections animales tout au long du processus de production et de collecte et réduire ces pertes. Le système d'élevage semi-intensif ou la claustration qui sont peu pratiqués dans la zone

\section{REMERCIEMENTS}

Les auteurs remercient la Direction du développement et de la coopération et le Fonds national Suisse pour la recherche scientifique dans le cadre du programme "Research on Global Issues for Development (R4D)" qui ont financé le projet IFWA (Sustainable use of insects to

\section{BIBLIOGRAPHIE}

Bayala J., Mando A., Teklehaimanot Z. et Ouédraogo SJ. 2005. Nutrient release from decomposing leaf mulches of karité (Vitellaria paradoxa) and néré (Parkia biglobosa) under semi-arid conditions in Burkina Faso, West Africa. Soil Biology \& Biochemistry, 37, 533-539

Bationo A., Kihara J., Vanlauwe B., Waswa B., Kimeu J. 2007. Soil organic carbon dynamics, functions and management in West African agroecosystems. Agric. Syst., 94 (1) : 13-25.

Berger M., Belem P.C., Dakouo D. \& Hien V., 1987. Le maintien de la fertilité des sols dans l'Ouest du Burkina Faso et la nécessité de l'association agriculture-élevage. Coton Fibres Trop., 42, 201210.

Berger M. 1996. L'amélioration de la fumure organique en Afrique soudano-sahélienne. Agriculture et développement (hors-série). Montpellier : Cirad.

Blanchard M, Coulibaly K, Bognini S, Dugué P, Vall E, 2014. Diversité de la qualité des fumures organiques produites par les paysans d'Afrique de l'Ouest: quelles conséquences sur les recommandations de fumure? Biotechnol. Agron. Soc. Environ. 18(4) : 512-523

Blanchard M., Coulibaly D., Ba A., Sissoko F., PoccardChappuis R., 2012. Contribution de l'intégration agriculture-élevage à l'intensification écologique des systèmes agro-sylvo-pastoraux : le cas du Mali-Sud. Vall E., Andrieu N., Chia E., Nacro H B. Partenariat, modélisation, expérimentations : quelles leçons pour la conception de l'innovation et l'intensification écologique ?, Nov. 2011, d'étude, augurent de bonnes perspectives pour améliorer non seulement la production de volaille mais aussi celle de la production de fumure organique avec respectivement 5,08 et $19,21 \mathrm{~kg} / \mathrm{sujet}$ de litière de volaille contre $4,67 \mathrm{~kg} / \mathrm{sujet}$ pour le système extensif. Un travail important dans l'alimentation de la volaille telle que l'introduction des asticots comme source protéique, dans ces systèmes améliorés d'élevage sera nécessaire.

improve livestock production and food security in smallholder farms in West Africa). Ils adressent également leurs sincères remerciements aux producteurs des villages d'études qui ont accepté répondre aux questions.

Bobo-Dioulasso, Burkina Faso. Cirad, 12 p., 2012, Colloques. <hal-00718658>

Dao N., 2016. Inventaire et optimisation des techniques de collecte et de production de termites dans trois régions du Burkina Faso : Centre Ouest, Plateau Central et Nord. Mémoire de fin de cycle. Université Polytechnique de BoboDioulasso, Burkina Faso, 67p.

Diawara M., 2013. Impact the l'Utilisation des Termites en Aviculture Traditionnelle au Burkina Faso. Mémoire de fin de cycle. Université Polytechnique de Bobo-Dioulasso, Burkina Faso, 43p.

Dugué, P., 1989. Possibilités et limites de l'intensification des systèmes de culture vivriers en zone Soudano-Sahélienne. Le cas du Yatenga (Burkina Faso).Thèse en Sciences agronomiques, Université de Montpellier, France, $269 \mathrm{p}$.

FAO, 2002. The Bangladesh model and other experiences in family poultry development. International Network for Family Poulty Development. Electronic conference, May 2002.

Fotsa JC, Rognon X, Tixier-Boichard $M$, Ngou Ngoupayou J D, Pone Kamdem D, Manjeli Y, Bordas A, 2007. Exploitation de la poule locale (Gallus gallus) en zone de forêt humide $d u$ Cameroun. Bulletin de Santé et de Production Animales en Afrique, 55, 59-73.

Gomgnimbou A., Coulibaly K., Sanon A., Bacyé B., Nacro B.H., Sédogo P.M. 2016. Study of the Nutrient Composition of Organic Fertilizers in the 
Zone of Bobo-Dioulasso (Burkina Faso). Int. Jour. Sci. Res. Sci. Eng. Tech. 2(4), 617-622.

Hardouin J, 1986. Mini-Élevage et sources méconnues de protéines animales. Annales de Gembloux, 92, 153-162.

Hardouin J., 2003. Production d'insectes à des fins économiques ou alimentaires : Mini-élevage et BEDIM. Notes fauniques de Gembloux, 50, pp $15-25$

Kondombo S. R. Improvement of village chicken production in a mixed (chicken-ram) farming system. PhD Thesis, Wageningen Institute of Animal Science, 2005, Wageningen University, The Netherlands.

Koulibaly B., Dakuo D., Ouattara A., Traoré O., Lompo F., Zombré P. N., Yao-Kouamé A. 2015. Effets de l'association du compost et de la fumure minérale sur la productivité d'un système de culture à base de cotonnier et de maïs au Burkina Faso. Tropicultura, 33 (2) : 125-134

Landais, E., Lhoste, P., 1990. L'association agricultureélevage en Afrique intertropicale : un mythe techniciste confronté aux réalités de terrain. Cahiers des Sciences Humaines, 217-235.

Lhoste P., 2004. Les relations agriculture-élevage. Oléagineux, corps gras, Lipides, 11 (4) http://dx.doi.org/10.1051/ocl.2004.0253

MRA., 2009. Politique de développement de l'élevage au Burkina Faso 2010-2020. Ouagadougou, Burkina Faso, 45p.

Ouédraogo B., Gnanda I. B., Sanfo R., Zoundi S. J. et Bayala B., 2015. Étude comparative des performances réalisées avec l'incorporation de la farine de coproduits de volaille et la farine des asticots dans des rations de poulets de chair au Burkina Faso. Revue Ivoirienne des Sciences et Technologies, 148 - 161, http://www.revist.ci:
Pousga S. et Boly H., 2009. Synthèse des travaux de recherche en aviculture au Burkina Faso: Rapport de recherche No 4. Réseau International pour le Développement de l'Aviculture Familiale, 18(1/2), 28 -35.

Pousga S. Supplementation strategies for semiscavenging chickens in Burkina Faso, Evaluation of some local feed resources. Doctoral Thesis, Swedish university of agricultural science (SLU). $2007 ; 116$ pp.

Sawadogo H., Bock L., Lacroix D. \& Zombré N.P., 2008. Restauration des potentialités de sols dégradés à l'aide du zaï et du compost dans le Yatenga (Burkina Faso), Biotechnol. Agron. Soc. Environ., 12, 3, 279-290.

Schipper L.A. et Sparling G.P., 2000. Performance of soil condition indicators across taxonomic groups and land uses. Soil Sci. Soc. of Ame. J., 64, 300-311.

Vall E., Koutou M., Blanchard M., Coulibaly K., Diallo M.A., et Andrieu N. 2012. Intégration agricultureélevage et intensification écologique dans les systèmes agro-sylvo-pastoraux de l'Ouest du Burkina Faso, province du Tuy. In : Vall E., Andrieu N., Chia E., Nacro H B. Partenariat, modélisation, expérimentations : quelles lécons pour la conception de l'innovation et l'intensification écologique?, Nov. 2011, BoboDioulasso, Burkina Faso. Cirad, 13 p., 2012, Colloques. <hal-00718613>

Vall E., Dugué P., Blanchard M., 2006. Le tissage des relations agriculture-élevage au fil du coton, 1990-2005, Cahiers Agriculture, 15 (1) : 72-79.

Zoumana C. \& César J., 1994. Production et utilisation du fumier dans la zone dense de Korhogo en Côte d'Ivoire. Agric. Syst. Africa, 4(2), 20-27. 